\title{
Generic Invertibility of Multidimensional FIR Filter Banks and MIMO Systems
}

\author{
Ka L. Law, Robert M. Fossum, Member, IEEE, and Minh N. Do, Senior Member, IEEE,
}

\begin{abstract}
We study the invertibility of $M$-variate Laurent polynomial $N \times P$ matrices. Such matrices represent multidimensional systems in various settings such as filter banks, multiple-input multiple-output systems, and multirate systems. Given an $N \times P$ Laurent polynomial matrix $\boldsymbol{H}\left(z_{1}, \ldots, z_{M}\right)$ of degree at most $k$, we want to find a $P \times N$ Laurent polynomial left inverse matrix $G(z)$ of $H(z)$ such that $G(z) H(z)=I$. We provide computable conditions to test the invertibility and propose algorithms to find a particular inverse.

The main result of this paper is to prove that $\boldsymbol{H}(\boldsymbol{z})$ is generically invertible when $N-P \geq M$; whereas when $N-P<$ $M$, then $\boldsymbol{H}(\boldsymbol{z})$ is generically noninvertible. As a result, we propose an algorithm to find a particular inverse of a Laurent polynomial matrix that is faster than current algorithms known to us.
\end{abstract}

Index Terms-Left Invertibility, Perfect Reconstruction, Gröbner Bases, Multidimensional Multirate Systems, Generic Property.

\section{INTRODUCTION}

During the last two decades, one dimensional multirate systems in digital signal processing have been thoroughly developed. Due to the high demand of multidimensional processing including image and video processing, volumetric data analysis, and spectroscopic imaging, multidimensional multirate systems require more extensive study. Perfect reconstruction, which guarantees that an original input can be perfectly reconstructed from the outputs, is one key property of a multidimensional multirate system.

In a multidimensional multirate system, a digital signal is split into several channels and processed with different sampling rates. The most popular multirate systems are filter banks shown in Fig. 1(a). In the analysis part, a digital input signal is filtered and then downsampled, generating multiple outputs at the lower rates. In the synthesis part, the multiple outputs are upsampled and then filtered to reconstruct the

Copyright (c) 2008 IEEE. Personal use of this material is permitted. However, permission to use this material for any other purposes must be obtained from the IEEE by sending a request to pubs-permissions@ieee.org.

K. L. Law was with the Department of Mathematics and the Coordinated Science Laboratory, University of Illinois at Urbana-Champaign, Urbana IL 61801. He is now with the Department of Communication Systems, Technische Universität, Darmstadt 64283, Germany (email: klaw@nt.tu-darmstadt.de).

R. M. Fossum was with the Department of Mathematics and the Beckman Institute, University of Illinois at Urbana-Champaign, Urbana IL 61801 (email: rmfossum@illinois.edu).

M. N. Do is with the Department of Electrical and Computer Engineering, the Coordinated Science Laboratory, and the Beckman Institute, University of Illinois at Urbana-Champaign, Urbana IL 61801 (email: minhdo@illinois.edu).

The partial results of this paper have been presented in ICASSP'09, Taipei, Taiwan, April 2009. original signal. Using the polyphase representation in the $z$ domain [1], [2], we can represent the analysis part as an $N \times P$ matrix $\boldsymbol{H}(\boldsymbol{z})$ (shown in Fig.1(b)) with entries in a Laurent polynomial ring $\mathbb{C}\left[z_{1}, z_{2}, \ldots, z_{M}, z_{1}^{-1}, \ldots, z_{M}{ }^{-1}\right]$. In this case $M$ is the dimension of signals, $N$ is the number of channels in the filter bank, and $P$ is the sampling factor at each channel. An application of this setting may arise in multichannel acquisition. In such an application we collect data about an unknown multidimensional signal $\boldsymbol{X}(\boldsymbol{z})$ as output of the analysis part in Fig. 1(a). The acquisition system (filters $H_{i}(\boldsymbol{z})$ and sampling matrix $\boldsymbol{D}$ ) is fixed and known beforehand. The objective is to reconstruct $\boldsymbol{X}(\boldsymbol{z})$ with a synthesis part $\boldsymbol{G}(\boldsymbol{z})$. The existence of a synthesis part becomes a purely mathematical question.

Therefore, our first problem is to consider whether there exists a $P \times N$ matrix $\boldsymbol{G}(\boldsymbol{z})$ over a Laurent polynomial ring $\mathbb{C}\left[z_{1}, z_{2}, \ldots, z_{M}, z_{1}^{-1}, \ldots, z_{M}^{-1}\right]$ for which $\boldsymbol{G}(\boldsymbol{z}) \boldsymbol{H}(\boldsymbol{z})=\boldsymbol{I}_{P}$ where $\boldsymbol{I}_{P}$ is the $P \times P$ identity matrix.

One dimensional perfect reconstruction finite impulse response (FIR) filter banks have been investigated in several studies [3], [4], [5]. The Euclidean algorithm plays a key role in the matrix inverse problem for one dimensional perfect reconstruction FIR filter banks [4] since it can be used to find the GCD of a family of polynomials. For multivariate polynomials, there is a GCD (since the ring is a unique factorization domain) but the GCD is not necessarily a linear combination of the polynomials. The theory of Gröbner bases has been introduced to compute with multivariate polynomials [6], [7] and the theory is widely used in multidimensional signal processing [8], [9], [10], [11], [12], [13]. Methods using Gröbner bases techniques for testing the invertibility of and for computing a particular inverse of an $N \times 1$ multivariate polynomial matrix $\boldsymbol{H}(\boldsymbol{z})$ were proposed in [14], [15]. For an $N \times P$ multivariate polynomial matrix $\boldsymbol{H}(\boldsymbol{z})$ where $P>1$, adjoint matrix methods are employed in [14], [16]. Park in [17] provides a method to find the inverse of a Laurent polynomial matrix $\boldsymbol{G}(\boldsymbol{z})$. His method involves transforming Laurent polynomials into polynomials by multiplying by a series of elementary matrices. In this paper, we offer a simpler and more direct algorithm to compute a particular Laurent polynomial inverse. We can then generate all inverses from a particular inverse. In this set of inverses, one find an optimal set of synthesis filters according to some design criteria [11], [15], [18].

The second problem is: When is the probability for the existence of an inverse for a given system high? Rajagopal and Potter [14] and Zhou and Do [19] have investigated this problem and made several conjectures. We investigated systems by varying $M, N$ and $P$. In experiments, we found 


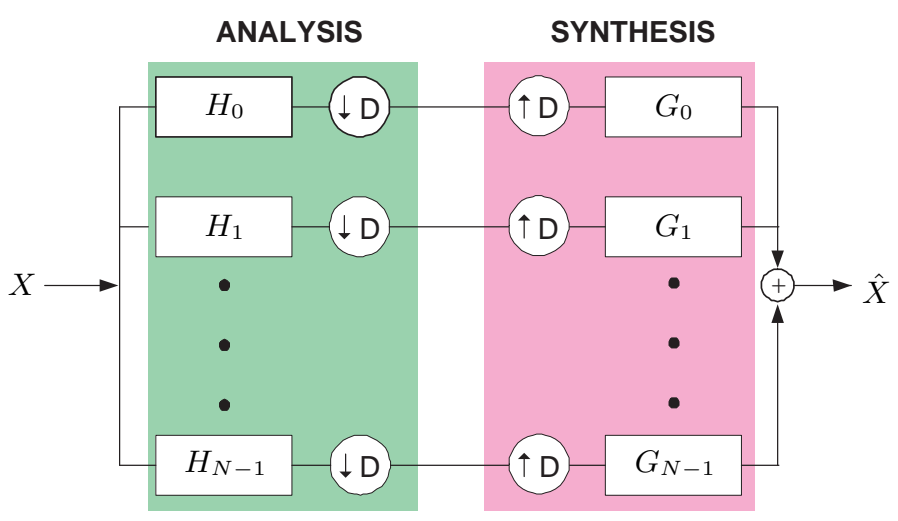

(a)

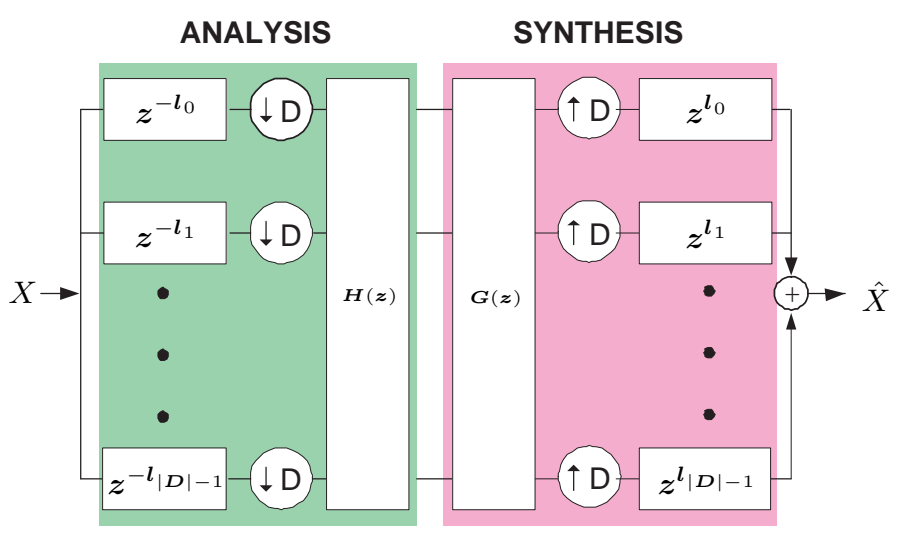

(b)

Fig. 1. Example system represented by a polynomial matrix. (a) A multidimensional $N$-channel oversampled filter bank: $H_{i}$ and $G_{i}$ are analysis and synthesis filters, respectively; $\boldsymbol{D}$ is an $M \times M$ sampling matrix with sampling rate $P=|\operatorname{det} \boldsymbol{D}| \leq N$. (b) Polyphase representation: $\boldsymbol{H}(\boldsymbol{z})$ and $\boldsymbol{G}(\boldsymbol{z})$ are analysis and synthesis polyphase transformation matrices, respectively; $\left\{\boldsymbol{l}_{i}\right\}$ is a basis of the lattice generated by the sampling matrix $\boldsymbol{D}$.

that when $M-N \geq P$, an inverse "almost surely" exists. On the other hand, when $M-N<P$, an inverse "almost surely" does not exist. To make precise the study of this inverse existence problem, we employ measure theory [20] and the concept of "holds generically" [7].

The paper is organized as follows. In Section II, we show how to verify the invertibility of a Laurent polynomial matrices. In Section III, we propose algorithms to find a particular inverse based on the Gröbner bases computation. Next, we characterize the set of all inverses. In Section IV, we prove that when $N-P \geq M$, then a polynomial matrix of degree at most $k$ is generically polynomial (resp.: Laurent polynomial) left invertible; whereas when $N-P<M$, then a polynomial matrix of degree at most $k$ is generically polynomial (resp.: Laurent polynomial) noninvertible. Based on this result, we present a fast algorithm to find a particular inverse in Section V. We conclude with a summary in Section VI.

\section{Mathematical CONTEXTS}

\section{A. (Left) Inverse Polynomial Matrix Problem}

We use boldface letters to denote vectors, or matrices. Let $\boldsymbol{z}$ be an $M$-dimensional complex variable $\boldsymbol{z}=\left(z_{1}, \ldots, z_{M}\right)$ in $\mathbb{C}^{M}$. For $\boldsymbol{n}=\left(n_{1}, \ldots, n_{M}\right) \in \mathbb{Z}^{M}$, we define the monomial $z^{n}=\prod_{i=1}^{M} z_{i}^{n_{i}}$. In this paper, we will always assume that $N, P$, and $M$ are positive integers.

Definition 1 (Polynomial or Laurent Polynomial Matrix): An $N \times P$ matrix $\boldsymbol{H}(\boldsymbol{z})$ is said to be a polynomial matrix (resp.: Laurent polynomial matrix) if every entry is a polynomial (resp.: Laurent polynomial).

Definition 2 (Left Invertible): An $N \times P$ polynomial (resp.: Laurent polynomial) matrix $\boldsymbol{H}(\boldsymbol{z})$ is said to be polynomial (resp.: Laurent polynomial) left invertible if there exists a $P \times$ $N$ polynomial (resp.: Laurent polynomial) matrix $\boldsymbol{G}(\boldsymbol{z})$ such that

$$
\boldsymbol{G}(\boldsymbol{z}) \boldsymbol{H}(\boldsymbol{z})=\boldsymbol{I}_{P}
$$

Otherwise $\boldsymbol{H}(\boldsymbol{z})$ is said to be polynomial (resp.: Laurent polynomial) left noninvertible. 
The discussion of polynomial (resp.: Laurent polynomial) left invertible can also apply to polynomial (resp.: Laurent polynomial) right invertible. To avoid repetition, throughout the paper we use the word "invertible" to represent either polynomial left invertible or Laurent polynomial left invertible. It will be clear in the context whether it is polynomial left invertible or Laurent polynomial left invertible. We will also restrain from using the pedantic "(resp.: Laurent polynomial)" when it is understood in the context.

Consider an $N \times 1$ matrix $H(z)$ over $\mathbb{C}[z]$ where $H_{i}(z)$ is the $i$-th row of $H(z)$. If the greatest common divisor (GCD) of $\left\{H_{1}(z), \ldots, H_{N}(z)\right\}$ is 1 , then the Bezout identity problem has a solution [21]. We can use the Euclidean algorithm to find the GCD and also a set of polynomials $\left\{G_{1}(z), \ldots, G_{N}(z)\right\}$ [22] such that

$$
\sum_{j=1}^{N} G_{j}(z) H_{j}(z)=1 \text {. }
$$

However, the univariate GCD criterion and Euclidean algorithm fail for multivariate polynomials. But the multivariate membership problem can be solved by using Gröbner bases [6], [23]. Briefly, the theory of Gröbner bases implies that any set of generators of an ideal or module has a unique reduced Gröbner basis for a given ordering. This basis is obtained by using Buchberger's algorithm [24].

In particular, suppose $\left\{\boldsymbol{b}_{1}(\boldsymbol{z}), \ldots, \boldsymbol{b}_{n}(\boldsymbol{z})\right\}$ is a Gröbner basis of a $\mathbb{C}[\boldsymbol{z}]$-submodule $\left\langle\boldsymbol{r}_{1}(\boldsymbol{z}), \ldots, \boldsymbol{r}_{N}(\boldsymbol{z})\right\rangle$ generated by $\boldsymbol{r}_{1}(\boldsymbol{z}), \ldots, \boldsymbol{r}_{N}(\boldsymbol{z})$ [25] where $\boldsymbol{r}_{i}(\boldsymbol{z})$ belongs to $\mathbb{C}[\boldsymbol{z}]^{P}$. Then there exists an $n \times N$ transformation matrix $\left\{W_{i j}(\boldsymbol{z})\right\}$ such that

$$
\boldsymbol{b}_{i}(\boldsymbol{z})=\sum_{j=1}^{N} W_{i j}(\boldsymbol{z}) \boldsymbol{r}_{j}(\boldsymbol{z}) \text {. }
$$

Buchberger's algorithm is implemented in most computer algebra software systems, such as Singular, Macauley2, Maple, and Mathematica, and hence the computation of Gröbner bases is available in these systems.

\section{B. Criteria for Left Invertibility}

To conclude a general fact between the Gröbner bases and invertibility of a polynomial matrix, we have the following proposition. This generalizes Proposition 2 from [15].

Proposition 1: Suppose $\boldsymbol{H}(\boldsymbol{z})$ is an $N \times P$ polynomial matrix. Let $S=\left\langle\boldsymbol{h}_{1}(\boldsymbol{z}), \ldots, \boldsymbol{h}_{N}(\boldsymbol{z})\right\rangle$ be the $\mathbb{C}[\boldsymbol{z}]$-submodule of $\mathbb{C}[\boldsymbol{z}]^{P}$ generated by the rows $\boldsymbol{h}_{i}(\boldsymbol{z})$ of $\boldsymbol{H}(\boldsymbol{z})$. Then $\boldsymbol{H}(\boldsymbol{z})$ is invertible if and only if the reduced Gröbner basis of $S$ is $\left\{\boldsymbol{e}_{i}\right\}_{i=1, \ldots, P}$ where $\boldsymbol{e}_{i}$ is the $i$-th row of the $P \times P$ identity matrix.

Proof: Suppose $\boldsymbol{H}(\boldsymbol{z})$ is invertible. Then there exist $\boldsymbol{G}(\boldsymbol{z})=\left(g_{i j}(\boldsymbol{z})\right)$ such that

$$
G(z) H(z)=I .
$$

Then

$$
\boldsymbol{e}_{i}=\sum_{j=1}^{N} g_{i j}(\boldsymbol{z}) \boldsymbol{h}_{j}(\boldsymbol{z})
$$

for $i=1, \ldots, P$. According to the definition of a Gröbner basis [6, p.121], $\left\{\boldsymbol{e}_{i}\right\}_{i=1, \ldots, P}$ is a Gröbner basis of $S$. It is a reduced Gröbner basis since the $\boldsymbol{e}_{i}$ are linearly independent. By the uniqueness of reduced Gröbner basis with respect to a given term order, $\left\{\boldsymbol{e}_{i}\right\}_{i=1, . ., P}$ is the reduced Gröbner basis of $S$.

Suppose on the other hand that the reduced Gröbner basis of $S$ is $\left\{\boldsymbol{e}_{i}\right\}_{i=1, . ., P}$. Then there exist some $\left\{g_{i j}(\boldsymbol{z})\right\}$ satisfying (3). Let $\boldsymbol{G}(\boldsymbol{z})=\left(g_{i j}(\boldsymbol{z})\right)$. Then

$$
\boldsymbol{G}(\boldsymbol{z}) \boldsymbol{H}(\boldsymbol{z})=\boldsymbol{I} .
$$

Thus $\boldsymbol{H}(\boldsymbol{z})$ is invertible.

Example 1: Is $\boldsymbol{H}\left(z_{1}, z_{2}\right)=\left(\begin{array}{cc}1 & 3 z_{2} \\ 2 z_{1}+1 & 0 \\ 3 & z_{1} \\ 3 z_{2} & 5\end{array}\right)$ invertible? We can use the software Singular [26] to implement the above result.

1: >ring $\mathrm{R}=0,(\mathrm{z}(1), \mathrm{z}(2)), \mathrm{dp} ;$ $R$ is a ring with 2 variables; dp specifies degree reverse lexicographical ordering

2: >matrix H[4] [2] ;

$3:>\mathrm{H}=1,3 \star \mathrm{z}(2), 2 \star \mathrm{z}(1)+1,0,3, \mathrm{z}(1), 3 \star \mathrm{z}(2), 5$;

4: $>$ print $(\mathrm{H})$;

5: $1,3 \star z(2)$,

6: $2 \star z(1)+1,0$,

7: $3, z(1)$,

8: $3 \star z(2), 5$

9: >module $\mathrm{S}=$ transpose $(\mathrm{H})$; $\quad \triangleright \mathrm{S}$ is the module generated by rows of $\boldsymbol{H}\left(z_{1}, z_{2}\right)$

10: >option (redSB); $\triangleright$ Computes a reduced standard basis in any standard basis computation

11: >print (std $(S))$; $\triangleright$ Returns the reduced Groebner basis by using above option

12: 1,0 ,

13: 0,1

By Proposition 1, we know that $\boldsymbol{H}\left(z_{1}, z_{2}\right)$ is invertible.

The results from algebraic geometry and Gröbner bases deal only with polynomial matrices. To be applicable for systems with general FIR filters, not just causal or anticausal filters, we need to extend the results from polynomial matrices to Laurent polynomial matrices. One method is to multiply both sides of (1) with a monomial of high enough degree. Thus $\boldsymbol{H}(\boldsymbol{z})$ is Laurent polynomial left invertible if and only if there exist a $P \times N$ polynomial matrix $\hat{\boldsymbol{G}}(\boldsymbol{z})$ such that

$$
\hat{\boldsymbol{G}}(\boldsymbol{z}) \boldsymbol{H}(\boldsymbol{z})=\boldsymbol{z}^{k} \boldsymbol{I}_{P}
$$

for some integer vector $\boldsymbol{k}$. But finding a suitable integer vector $k$ might require an extensive search. However, by generalizing Theorem 2 from [15], we have a simple algorithm to determine whether the given Laurent polynomial matrix is invertible or not $^{1}$.

Proposition 2: Suppose $\boldsymbol{H}(\boldsymbol{z})$ is an $N \times P$ Laurent polynomial matrix. Consider the $(N+P) \times P$ matrix

$$
\boldsymbol{H}^{\prime}(\boldsymbol{z}, w)=\left(\begin{array}{c}
\boldsymbol{z}^{\boldsymbol{m}} \boldsymbol{H}(\boldsymbol{z}) \\
\operatorname{diag}\left(1-z_{1} z_{2} \ldots z_{M} w\right)
\end{array}\right)
$$

${ }^{1}$ Theorem 2 from [15] can be proved directly by using the "Rabinowitch trick". See also [27]. 
where $\boldsymbol{m} \in \mathbb{N}^{M}$ is such that $\boldsymbol{z}^{\boldsymbol{m}} \boldsymbol{H}(\boldsymbol{z})$ is a polynomial matrix, $w$ is a new variable, and $\operatorname{diag}(y)$ is a $P \times P$ diagonal matrix with element $y$ on the diagonal. Then $\boldsymbol{H}(\boldsymbol{z})$ is Laurent polynomial left invertible if and only if $\boldsymbol{H}^{\prime}(\boldsymbol{z}, w)$ is a polynomial left invertible matrix.

Proof: If $\boldsymbol{H}(\boldsymbol{z})$ is Laurent polynomial left invertible, then $\boldsymbol{z}^{\boldsymbol{m}} \boldsymbol{H}(\boldsymbol{z})$ is also Laurent polynomial left invertible. Then there exists a polynomial matrix $\boldsymbol{G}(\boldsymbol{z})=\left(g_{i j}(\boldsymbol{z})\right)$ satisfying (4). Among these $\boldsymbol{k}$, pick one for which $\boldsymbol{m}^{\prime} \in \mathbb{Z}_{+}^{\mathbb{M}}$ is the least integer vector. Let $m_{0}$ be the maximal entry of $\boldsymbol{m}^{\prime}=$ $\left\{m_{1}, \ldots, m_{M}\right\}$. If $m_{0}=0$, then $\boldsymbol{H}(\boldsymbol{z})$ is polynomial left invertible and so is $\boldsymbol{H}^{\prime}(\boldsymbol{z}, w)$. Otherwise $m_{0}$ is positive. Now let

$g_{i j}^{\prime}(\boldsymbol{z}, w)= \begin{cases}w^{m_{0}} \prod_{k=1}^{M} z_{k}^{m_{0}-m_{k}} g_{i j}(\boldsymbol{z}), & i=1, \ldots, P ; j=1, \ldots, N ; \\ \sum_{k=0}^{m_{0}-1}\left(\prod_{l=1}^{M} z_{l}^{k}\right) w^{k}, & \text { if } i=j-N ; \\ 0, & \text { otherwise. }\end{cases}$

Let $\boldsymbol{G}^{\prime}(\boldsymbol{z}, w)=\left(g_{i j}^{\prime}(\boldsymbol{z}, w)\right)$ be the corresponding $P \times(P+N)$ matrix. Then by a straightforward computation, we can conclude that $\boldsymbol{G}^{\prime}(\boldsymbol{z}, w)$ is a polynomial left inverse of $\boldsymbol{H}^{\prime}(\boldsymbol{z}, w)$.

Now suppose $\boldsymbol{H}^{\prime}(\boldsymbol{z}, w)$ is polynomial left invertible. There exists $\boldsymbol{G}^{\prime}(\boldsymbol{z}, w)$ such that $\boldsymbol{G}^{\prime}(\boldsymbol{z}, w) \boldsymbol{H}^{\prime}(\boldsymbol{z}, w)=\boldsymbol{I}$ with $\boldsymbol{G}^{\prime}(\boldsymbol{z}, w)=\left(g_{i j}^{\prime}(\boldsymbol{z}, w)\right)$. Set

$$
\boldsymbol{G}(\boldsymbol{z})=\left(\boldsymbol{z}^{-\boldsymbol{m}} g_{i j}^{\prime}\left(\boldsymbol{z}, \prod_{k=1}^{M} z_{k}^{-1}\right)\right)_{i=1, \ldots, P ; j=1, \ldots, N} .
$$

Then we have $\boldsymbol{G}(\boldsymbol{z}) \boldsymbol{H}(\boldsymbol{z})=\boldsymbol{I}$ and $\boldsymbol{G}(\boldsymbol{z})$ is a Laurent polynomial matrix. Hence $\boldsymbol{H}(\boldsymbol{z})$ is Laurent polynomial left invertible.

Example 2: Is $\boldsymbol{H}(\boldsymbol{z})=\left(\begin{array}{cc}z_{1} & z_{1} \\ z_{2}^{2}+3 & z_{2}^{2}+1\end{array}\right)$ invertible? Clearly it is not polynomial invertible because the determinant is zero when $z_{1}$ is zero. To verify that the matrix is Laurent polynomial left invertible, we need to introduce a new variable and the $\boldsymbol{H}^{\prime}(\boldsymbol{z}, w)$ from (5) and test the invertibility of $\boldsymbol{H}^{\prime}(\boldsymbol{z}, w)$.

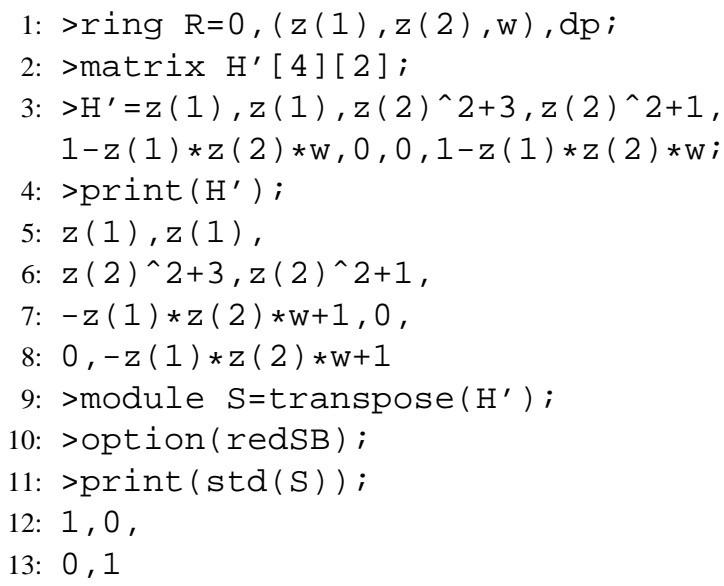

This implies that $\boldsymbol{H}(\boldsymbol{z})$ is Laurent polynomial left invertible.

\section{PROPOSED ALGORITHMS}

\section{A. Computation of Left Inverses}

In this section we introduce two new algorithms to generate an inverse matrix of a given matrix if the matrix is invertible.
These algorithms use Gröbner bases and are based on Proposition 1 and Proposition 2.

Algorithm 1 Particular Polynomial Inverse

The computational algorithm for a polynomial left inverse matrix

Input: $N \times P$ polynomial matrix $\boldsymbol{H}(\boldsymbol{z})$ over $\mathbb{C}\left[z_{1}, \ldots, z_{M}\right]$

Output: $P \times N$ polynomial matrix $\boldsymbol{G}(\boldsymbol{z})$, if it exists

1: compute the reduced Gröbner basis of $\left\{\boldsymbol{h}_{1}(\boldsymbol{z}), \ldots, \boldsymbol{h}_{N}(\boldsymbol{z})\right\}$ where $\boldsymbol{h}_{i}(\boldsymbol{z})$ is a row of $\boldsymbol{H}(\boldsymbol{z})$ and the associated transformation matrix $\left\{W_{i j}(\boldsymbol{z})\right\}$ as defined in (2)

2: if the reduced Gröbner basis is $\left\{\boldsymbol{e}_{i}\right\}_{i=1, \ldots, P}$, then output $\left(W_{i j}(\boldsymbol{z})\right)$

3: else there is no solution

: end if

Algorithm 2 Particular Laurent Polynomial Inverse
The computational algorithm for a Laurent polynomial left
inverse matrix
Input: $N \times P$ Laurent polynomial matrix $\boldsymbol{H}(\boldsymbol{z})$ with $M$
variables
Output: $P \times N$ Laurent polynomial matrix $\boldsymbol{G}(\boldsymbol{z})$, if it exists
1: multiply $\boldsymbol{H}(\boldsymbol{z})$ by a common monomial $\boldsymbol{z}^{\boldsymbol{m}}$ such that
$\quad \boldsymbol{H}^{\prime}(\boldsymbol{z}, w)$ is polynomial matrix from Proposition 2
2: call Algorithm 1 with input $\boldsymbol{H}^{\prime}(\boldsymbol{z}, w)$
3: if the output of Algorithm 1 is $\boldsymbol{G}^{\prime}(\boldsymbol{z}, w)$, then output
$\quad \boldsymbol{z}^{-\boldsymbol{m}}\left(\boldsymbol{G}_{i j}^{\prime}\left(\boldsymbol{z}, \prod_{k=1}^{M} z_{k}^{-1}\right)\right)_{i=1, \ldots, P ; j=1, \ldots, N}$
4: else there is no solution
5: end if

Example 3: Find an inverse of $\boldsymbol{H}\left(z_{1}, z_{2}\right)=$ $\left(\begin{array}{cc}1 & 3 z_{2} \\ 2 z_{1}+1 & 0 \\ 3 & z_{1} \\ 3 z_{2} & 5\end{array}\right)$. By Example 1 , we know that $\boldsymbol{H}\left(z_{1}, z_{2}\right)$ is invertible.

To calculate a left inverse of polynomial matrix, we have the following:

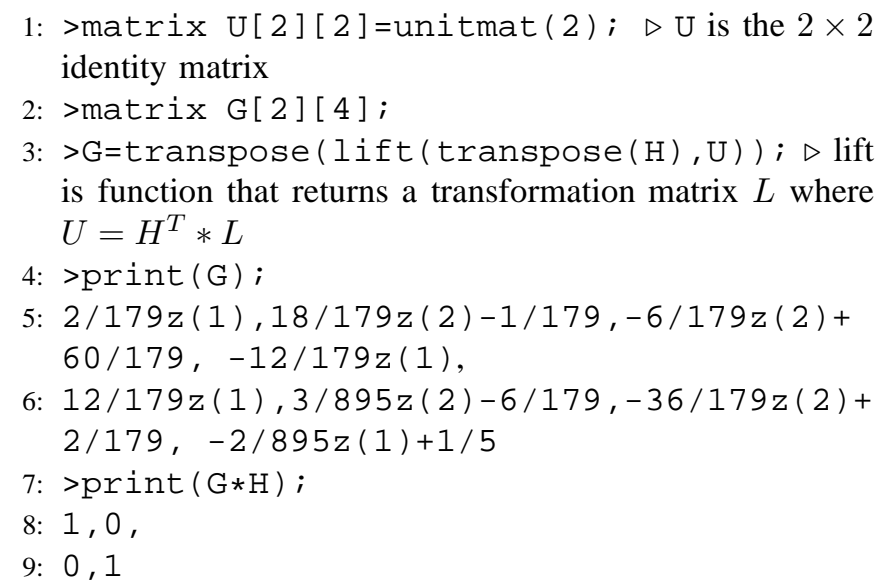

Thus $\boldsymbol{G}\left(z_{1}, z_{2}\right)$ is a left inverse of $\boldsymbol{H}\left(z_{1}, z_{2}\right)$.

Example 4: Find an inverse of $\boldsymbol{H}(\boldsymbol{z})=$ $\left(\begin{array}{cc}z_{1} & z_{1} \\ z_{2}^{2}+3 & z_{2}^{2}+1\end{array}\right)$. By Example 2, we know that $\boldsymbol{H}(\boldsymbol{z})$ is 
Laurent polynomial left invertible. To calculate a left inverse using Singular:

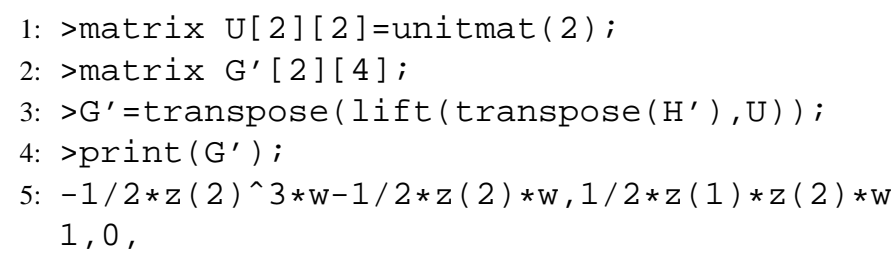

6: $1 / 2 \star z(2) \wedge 3 * w+3 / 2 \star z(2) \star w,-1 / 2 \star z(1) \star z(2) \star w$ 0,1

7: $>$ print $\left(\mathrm{G}^{\prime} \star \mathrm{H}^{\prime}\right)$;

8: 1,0 ,

9: 0,1

According to Algorithm 2, $\boldsymbol{G}(\boldsymbol{z}) \quad=$ $\left(\begin{array}{cc}-\frac{1}{2} z_{1}^{-1} z_{2}^{2}-\frac{1}{2} z_{1}^{-1} & \frac{1}{2} \\ \frac{1}{2} z_{1}^{-1} z_{2}^{2}+\frac{3}{2} z_{1}^{-1} & -\frac{1}{2}\end{array}\right)$ is a left inverse of $\boldsymbol{H}(\boldsymbol{z})$.

Rajagopal and Potter explore the computation of the synthesis part of an $M$-variate perfect reconstruction FIR filter. Their algorithm [14], [16] first computes every maximal minor of $\boldsymbol{H}(\boldsymbol{z})$ and the corresponding adjoint matrix. Then it uses them to compute an inverse of $\boldsymbol{H}(\boldsymbol{z})$. The size of the set of maximal minors is $\left(\begin{array}{l}N \\ P\end{array}\right)$, which could be large if $N-P$ is large. When this is the case, we find in practice that the algorithm is extremely slow. In order to avoid the problem that the computation of maximal minors poses, our Algorithm 2 computes an inverse directly by using the computation of the reduced Gröbner bases for modules. Park [17] also presents an algorithm. To find the inverse of Laurant polynomial matrices, Park transforms the Laurent polynomial matrix into a polynomial matrix by multiplying by a series of elementary matrices. Our approach simply transforms Laurent polynomials into polynomials by multiplying by a large enough monomial. Therefore our approach is simpler and provides a closed form formula to compute an inverse.

When one designs a filter bank, one would like to estimate the degree of the entries in the inverse matrices. Caniglia et al. [28] propose an upper bound on the degree of $N \times N$ invertible matrix $\boldsymbol{K}(\boldsymbol{z})$ such that $\boldsymbol{K}(\boldsymbol{z}) \boldsymbol{H}(\boldsymbol{z})=\left[\begin{array}{c}\boldsymbol{I}_{P} \\ \mathbf{0}\end{array}\right]$ and the degree bound of $\operatorname{deg}(\boldsymbol{K}(\boldsymbol{z}))$ is optimal in order.

Proposition 3: [28] Assume that $\boldsymbol{H}(\boldsymbol{z})$ is an $N \times P$ invertible matrix in $M$ variables. Let $\operatorname{deg}(\boldsymbol{H}(\boldsymbol{z}))$ be the maximum of the degrees of the entries of $\boldsymbol{H}(\boldsymbol{z})$ and let $d=\operatorname{deg}(\boldsymbol{H}(\boldsymbol{z}))+$ 1. Then there exists an $N \times N$ invertible matrix $\boldsymbol{K}(\boldsymbol{z})$ such that

$$
\boldsymbol{K}(\boldsymbol{z}) \boldsymbol{H}(\boldsymbol{z})=\left[\begin{array}{c}
\boldsymbol{I}_{P} \\
\mathbf{0}
\end{array}\right]
$$

and $\operatorname{deg}(\boldsymbol{K}(\boldsymbol{z}))$ is $(P d)^{O(M)}$.

This suggests that the maximum degree of the entries of the $P \times N$ inverse matrix $\boldsymbol{G}(\boldsymbol{z})$ is also less than or equal to $(P d)^{O(M)}$.

\section{B. Characterization of Inverses}

Algorithms 1 and 2 do not guarantee that the inverse would be well behaved. In this section, we refer to some results that characterize the set of all inverses. Once we have a particular inverse, we can parametrize the set of all inverses.
First we make a simple observation. Suppose $g, h$ are elements in some ring for which $g h=1$ while $h g \neq 1$. Then $h g h g=h g$ and $(1-h g) h=0$. If $a$ is any element, then $a(1-h g) h=0$ and hence

$$
(g+a(1-h g)) h=1 .
$$

Thus if $h g \neq 1$, then we can find infinitely many left inverses to $h$. With this in mind we have the following general atements.

Lemma 1 (Zhou): [19], [29] Suppose $\boldsymbol{H}(\boldsymbol{z})$ is an $N \times P$ polynomial matrix and $\tilde{\boldsymbol{G}}(\boldsymbol{z})$ is a $P \times N$ polynomial matrix such that $\tilde{\boldsymbol{G}}(\boldsymbol{z}) \boldsymbol{H}(\boldsymbol{z})=\boldsymbol{I}$. Then $\boldsymbol{G}(\boldsymbol{z})$ is an polynomial inverse matrix of $\boldsymbol{H}(\boldsymbol{z})$ if and only if $\boldsymbol{G}(\boldsymbol{z})$ can be written as

$$
\boldsymbol{G}(\boldsymbol{z})=\tilde{\boldsymbol{G}}(\boldsymbol{z})+\boldsymbol{A}(\boldsymbol{z})(\boldsymbol{I}-\boldsymbol{H}(\boldsymbol{z}) \tilde{\boldsymbol{G}}(\boldsymbol{z}))
$$

where $\boldsymbol{A}(\boldsymbol{z})$ is an arbitrary $P \times N$ polynomial matrix.

Theorem 1 (Park): [30] Suppose $\boldsymbol{H}(\boldsymbol{z})$ is an $N \times P$ polynomial matrix and $\tilde{\boldsymbol{G}}(\boldsymbol{z})$ is a $P \times N$ polynomial matrix such that $\tilde{\boldsymbol{G}}(\boldsymbol{z}) \boldsymbol{H}(\boldsymbol{z})=\boldsymbol{I}$. Let $\boldsymbol{h}_{1}, \boldsymbol{h}_{2}, \ldots, \boldsymbol{h}_{N}$ be row vectors of $\boldsymbol{H}(\boldsymbol{z})$. Then $\boldsymbol{G}(\boldsymbol{z})$ is an polynomial inverse matrix of $\boldsymbol{H}(\boldsymbol{z})$ if and only if $\boldsymbol{G}(\boldsymbol{z})$ can be written as

$$
\boldsymbol{G}(\boldsymbol{z})=\tilde{\boldsymbol{G}}(\boldsymbol{z})+\boldsymbol{A}(\boldsymbol{z}) \operatorname{Syz}\left(\boldsymbol{h}_{1}, \boldsymbol{h}_{2}, \ldots, \boldsymbol{h}_{N}\right)
$$

where $\boldsymbol{A}(\boldsymbol{z})$ is an arbitrary polynomial matrix and Syz is the syzygy [6] of $\left\{\boldsymbol{h}_{1}, \boldsymbol{h}_{2}, \ldots, \boldsymbol{h}_{N}\right\}$.

Remark 1: Both of these theorems hold when polynomial is replaced by Laurent polynomial.

Remark 2: Note that since $(\boldsymbol{I}-\boldsymbol{H}(\boldsymbol{z}) \tilde{\boldsymbol{G}}(\boldsymbol{z})) \boldsymbol{H}(\boldsymbol{z})=0$, the element $(\boldsymbol{I}-\boldsymbol{H}(\boldsymbol{z}) \tilde{\boldsymbol{G}}(\boldsymbol{z}))$ is a syzygy of $\left\{h_{1}, \ldots, h_{N}\right\}$.

Zhou's method provides a simple characterization of inverses which is easy to implement. Park's method is more complicated. However the matrix size of the free parameter $\boldsymbol{A}(\boldsymbol{z})$ in Lemma 1 is $P \times N$, while the smallest possible matrix size of $\boldsymbol{A}(\boldsymbol{z})$ in Theorem 1 is $P \times(N-P)$ in theory. Though syzygy provided by Singular does not necessary attain this optimal size, the matrix size for $\boldsymbol{A}(\boldsymbol{z})$ obtained in Park's method is in general smaller than Zhou's method.

Example 5: Let be $\boldsymbol{H}(\boldsymbol{z})=\left(\begin{array}{cc}z_{1} & z_{1}+1 \\ z_{2}+z_{1} & z_{1} \\ 3 & z_{1}+2 \\ z_{1} & z_{2}\end{array}\right)$. Find the

size of $\boldsymbol{A}\left(z_{1}, z_{2}\right)$ from Theorem 1 using Singular.

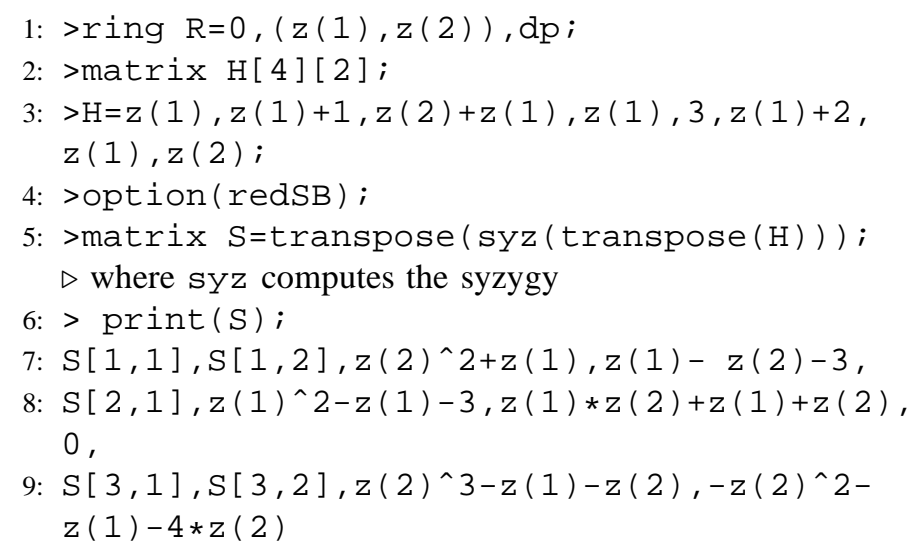

where $S[i, j]$ is some long polynomial expression. Thus the required free parameter $\boldsymbol{A}\left(z_{1}, z_{2}\right)$ in Theorem 1 is a $2 \times 3$ 
matrix. It is not the optimal matrix size, namely $2 \times 2$. But the size of $\boldsymbol{A}\left(z_{1}, z_{2}\right)$ in Zhou's method is $2 \times 4$. Therefore applying Park's method using Singular would lead to smaller size of $\boldsymbol{A}(\boldsymbol{z})$ in this case.

In the set of all inverses, an optimal set of synthesis filters can be obtained according to some design criteria [11], [15], [18].

\section{GENERIC INVERTIBILITY}

\section{A. Lebesgue Measure and Generic Property}

When designing filter banks, an important question is how likely it is that the synthesis part of the perfect reconstruction filter banks exists. If it does not exist, then in general we are not able to reconstruct the original signal.

In [19], Zhou and Do made the following conjecture.

Conjecture 1: Suppose $\boldsymbol{H}(\boldsymbol{z})$ is an $M$-variate $N \times P$ polynomial (resp.: Laurent polynomial) matrix with $N \geq P$. If $N-P \geq M$, then it is "almost surely" polynomial (resp.: Laurent polynomial) left invertible. Otherwise, it is "almost surely" polynomial' (resp.: Laurent polynomial) left noninvertible.

Rajagopal and Potter made another conjecture related to "almost surely" invertible in their paper [14].

Corollary 6 in [14]: Suppose $\boldsymbol{H}(\boldsymbol{z})$ is an $N \times P M$-variate polynomial matrix with $N>P$. If $\left(\begin{array}{l}N \\ P\end{array}\right)>M$, then it is "almost surely" invertible.

Unfortunately, Corollary 6 in [14] is not correct. Please refer to Zhou's thesis [29] for more details.

Suppose the conjecture posed by Zhou and Do is true. If we design filter banks such that $N-P \geq M$, then "almost surely" there exists a synthesis part of the filter banks which is able to reconstruct the original signal perfectly.

However, Zhou and Do did not give a precise definition of "almost surely". In order to have the appropriate language, we employ the concept of Lebesgue measure and the concept of "hold generically".

In the 2-dimensional plane, it is obvious that any "simple" line (i.e. not a locally space filling curve) has zero area. In 3dimensional space, we also know that any "simple" surface has zero volume. To generalize this property, we have the following lemma.

Lemma 2: [31, p.9] Let $f$ be holomorphic (which means infinitely differentiable) in the domain $D \subset \mathbb{C}^{M}$, and suppose $f$ is not identically zero. Then $\lambda_{M}(\{z \in D \mid f(z)=0\})=0$ where $\lambda_{M}$ denote the $2 M$-dimensional Lebesgue measure.

Definition 3 (Generic): [32] A property is said to hold generically for polynomials $f_{1}, \ldots, f_{n}$ of degree at most $k_{1}, \ldots, k_{n}$ if there is a nonzero polynomial $F$ in the coefficients of the $f_{i}$ such that the property holds for $f_{1}, \ldots, f_{n}$ whenever the polynomial $F\left(f_{1}, \ldots, f_{n}\right)$ is nonvanishing.

Intuitively, a property of polynomials is generic if it holds for "almost all" polynomials.

Example 6: [32] The property " $f(x)=c_{2} x^{2}+c_{1} x+c_{0}$ has two distinct solutions" is generic.

Proof: Let $F$ be a polynomial of the coefficients of $f=$ $c_{2} x^{2}+c_{1} x+c_{0}$ given by

$$
F=c_{2}\left(c_{1}^{2}-4 c_{2} c_{0}\right)
$$

Suppose $F(f)$ is nonzero (i.e. $c_{2}\left(c_{1}^{2}-4 c_{2} c_{0}\right) \neq 0$ ). Then $c_{2} \neq 0$ and $c_{1}^{2}-4 c_{2} c_{0} \neq 0$. So $f$ has two distinct solutions. Therefore by the above definition, $f(x)=c_{2} x^{2}+c_{1} x+c_{0}$ has two distinct solutions generically.

Lemma 3: If a property of polynomials of degree at most $k_{1}, \ldots, k_{n}$ in $M$ variables is generic, then the coefficient space $\mathcal{C}$ of polynomials whose polynomials failed to satisfy the property is measure zero and nowhere dense.

Proof: By the definition of hold generically, there exists a nonzero polynomial $F$ in the coefficients of the $f_{i}$ such that the property fails to satisfy for $f_{1}, \ldots, f_{n}$ for which the polynomial $F\left(f_{1}, \ldots, f_{n}\right)$ is vanishing. Let $R_{i}$ be the set of $M$-variate polynomials of degree less than or equal to $k_{i}$. By lemma 2,

$$
\lambda_{l}\left(\left\{\left(f_{1}, \ldots, f_{n}\right) \in \prod_{i=1}^{n} R_{i} \mid F\left(f_{1}, \ldots, f_{n}\right)=0\right\}\right)=0
$$

where $l=\left(\begin{array}{c}k_{1}+M \\ M\end{array}\right)+\ldots+\left(\begin{array}{c}k_{n}+M \\ M\end{array}\right)$ is the dimension of the coefficient space. Thus, the coefficient space $\mathcal{C}$ of polynomials whose polynomials failed to satisfy the property is measure zero. To show the set is nowhere dense, it is equivalent to show that the closure of the set contains no open set. Suppose it contains an open ball $B(\epsilon)$ with some radius $\epsilon>0$. Since $F^{-1}(\{0\})$ is a closed set, $\overline{\mathcal{C}}$ is also in $F^{-1}(\{0\})$. Thus, $F^{-1}(\{0\})$ contains the open ball $B(\epsilon)$. However, this contradicts the fact that $F^{-1}(\{0\})$ is measure zero. Therefore, the coefficient space of polynomials whose polynomials failed to satisfy the property is nowhere dense.

The immediate consequence is that if $f_{1}, \ldots, f_{n}$ are drawn independently from a probability distribution with respect to the Lebesgue measure, the property of $f_{1}, \ldots, f_{n}$ holds with probability one. Furthermore, suppose $\tilde{f}_{0}, \ldots, \tilde{f}_{n}$ satisfies the property. Since the coefficient space $\mathcal{C}$ of polynomials whose polynomials failed to satisfy the property is nowhere dense, there exists an open ball $B(\epsilon)$ around $\tilde{f}_{0}, \ldots, \tilde{f}_{n}$ for some $\epsilon>0$ such that the property is satisfied within the open ball $B(\epsilon)$. This shows that the system with the property is robust [33].

\section{B. Generically Invertible when $N-P \geq M$}

To prove our main theorem in this section, we need to employ the resultant of the polynomials.

Theorem 2 (Resultant): [7, p.80] If we fix positive degrees $k_{0}, \ldots, k_{n}$, then there is a unique nonzero polynomial called the resultant $\left.\operatorname{RES}_{\left(k_{0}, \ldots, k_{n}\right)} \in \mathbb{C}\left[\bigcup_{i=1}^{n}\left\{u_{i j}\right\}_{j=1, \ldots,\left({ }_{i}+M\right.}{ }_{M}\right)\right]$ where the variables $u_{i j}$ correspond to the coefficients of $i$-th polynomial. Then we have the following property:

If $F_{0}, \ldots, F_{n} \in \mathbb{C}\left[x_{0}, \ldots, x_{M}\right]$ are homogeneous of degrees $k_{0}, \ldots, k_{n}$, then $F_{0}, \ldots, F_{n}$ have a nontrivial common zero over $\mathbb{C}$ if and only if $\operatorname{RES}_{\left(k_{0}, \ldots, k_{n}\right)}\left(F_{0}, \ldots, F_{n}\right)=0$.

Now we can translate the first half of Conjecture 1 into the following mathematical framework.

Theorem 3: If $N-P \geq M$ and $k>0$, then an $N \times P$ polynomial $M$-variate matrix $\boldsymbol{H}(\boldsymbol{z})$ of degree at most $k$ is generically polynomial left invertible.

Proof: The strategy of this proof is to find a nonzero polynomial $F$ such that $F(\boldsymbol{H}(\boldsymbol{z}))=0$ for every noninvertible matrix $\boldsymbol{H}(\boldsymbol{z})$ of degree at most $k$. 
Let $\boldsymbol{Z}=\left(z_{0}, \ldots, z_{M}\right)$. If $f(\boldsymbol{z})=f_{0}(\boldsymbol{z})+f_{1}(\boldsymbol{z})+\ldots+f_{l}(\boldsymbol{z})$ is the decomposition of the polynomial $f(z)$ into sums of forms $f_{i}(\boldsymbol{z})$ of degree $i$, then the homogenization $\bar{f}(\boldsymbol{Z})$ of $f(\boldsymbol{z})$ of degree $k$ is defined to be $\bar{f}(\boldsymbol{Z})=z_{0}^{k} f_{0}(\boldsymbol{z})+z_{0}^{k-1} f_{1}(\boldsymbol{z})+$ $\ldots+z_{0}^{k-l} f_{l}(\boldsymbol{z})$. Let $\boldsymbol{h}_{i}(\boldsymbol{Z})$ be the $i$ th row of an $N \times P$ matrix $\overline{\boldsymbol{H}}(\boldsymbol{z})$. Let $t_{i-1}(\boldsymbol{Z})$ be the determinant of the $P \times P$ submatrix containing $\boldsymbol{h}_{i}(\boldsymbol{Z}), \boldsymbol{h}_{i+1}(\boldsymbol{Z}), \ldots, \boldsymbol{h}_{i+P-1}(\boldsymbol{Z})$. Define $\phi$ to be a function such that

$$
\boldsymbol{H}(\boldsymbol{z}) \mapsto\left(t_{0}(\boldsymbol{Z}), t_{1}(\boldsymbol{Z}), \ldots, t_{M}(\boldsymbol{Z})\right)^{T} .
$$

Rajagopal and Potter in [14], [16] show that if $\boldsymbol{H}(\boldsymbol{z})$ is noninvertible and $N \geq P$, then the $P \times P$ maximal minors of $\boldsymbol{H}(\boldsymbol{z})$ have a common zero. Suppose $\left(\tilde{z}_{1} / \tilde{z}_{0}, \tilde{z}_{2} / \tilde{z}_{0}, \ldots, \tilde{z}_{M} / \tilde{z}_{0}\right)$ is a solution of the maximal minors of $\boldsymbol{H}(\boldsymbol{z})$ where $\tilde{z}_{0} \neq 0$. Then $\left(\tilde{z}_{0}, \tilde{z}_{1}, \tilde{z}_{2}, \ldots, \tilde{z}_{M}\right)$ is a nonzero solution of maximal minors of $\overline{\boldsymbol{H}}(\boldsymbol{Z})$. Since $\left\{t_{0}, \ldots, t_{M}\right\}$ is a part of the subset of the set of maximal minors of $\overline{\boldsymbol{H}}(\boldsymbol{Z})$, this implies that $\phi(\boldsymbol{H}(\boldsymbol{z}))$ have a nontrivial common zero. Therefore, by the property of the resultant shown in Theorem 2, we know

$$
\operatorname{RES}_{(P k, \ldots, P k)}(\phi(\boldsymbol{H}(\boldsymbol{z})))=0
$$

for all noninvertible matrices $\boldsymbol{H}(\boldsymbol{z})$ of degree at most $k$. The $\operatorname{RES}_{(P k, \ldots, P k)}$ and $t_{i}$ are polynomials, so is $\operatorname{RES}_{(P k, \ldots, P k)} \circ \phi$. Last but not least, we need to show $\operatorname{RES}_{(P k, \ldots, P k)} \circ \phi$ is not a zero function. Let

$$
\boldsymbol{T}(\boldsymbol{z})=\left(\begin{array}{cccc}
1 & 0 & \ldots & 0 \\
z_{1}^{k} & 1 & \ldots & 0 \\
z_{2}^{k} & z_{1}^{k} & \ddots & 0 \\
\vdots & \vdots & \ddots & 1 \\
z_{M}^{k} & z_{M-1}^{k} & \ddots & z_{1}^{k} \\
0 & z_{M}^{k} & \ddots & \vdots \\
\vdots & \vdots & \ddots & \vdots \\
0 & \ldots & 0 & z_{M}^{k} \\
0 & \ldots & 0 & 0 \\
\vdots & \vdots & \vdots & \vdots \\
0 & \ldots & 0 & 0
\end{array}\right)
$$

be an $N \times P$ matrix. Suppose $\operatorname{RES}_{(P k, \ldots, P k)}(\phi(\boldsymbol{T}(\boldsymbol{z})))=0$. By Theorem 2, we know the $t_{i}$ 's have a nontrivial common zero. i.e. there exists $\tilde{Z}$ a nonzero solution such that

$$
t_{M}(\tilde{\boldsymbol{Z}})=\tilde{z}_{M}^{P k}=0 .
$$

This implies $\tilde{z}_{M}=0$. If $\tilde{z}_{M}=0$, then $t_{M-1}\left(\tilde{z}_{0}, \tilde{z}_{1}, \ldots, \tilde{z}_{M-1}, 0\right)=\tilde{z}_{M-1}^{P k}=0$. Thus $\tilde{z}_{M-1}=0$. Continuing the process, we can conclude $\tilde{z}_{0}=\tilde{z}_{1}=\ldots=\tilde{z}_{M}=0$. This contradicts the assumption that $\tilde{\boldsymbol{Z}}$ is nontrivial. So $\operatorname{RES}_{(P k, \ldots, P k)}(\phi(\boldsymbol{T}(\boldsymbol{z}))) \neq 0$. Therefore $\operatorname{RES}_{(P k, \ldots, P k)} \circ \phi$ is not the zero function. By the definition of hold generically, we conclude that $\boldsymbol{H}(\boldsymbol{z})$ of degree at most $k$ is generically polynomial left invertible matrix.

By multiplying a large enough common monomial, it is sufficient to consider only polynomial matrices.

Theorem 4: If $N-P \geq M$ and $k>0$, then an $N \times P$ polynomial $M$-variate matrix $\boldsymbol{H}(\boldsymbol{z})$ of degree at most $k$ is generically Laurent polynomial left invertible.
Proof: We know that if a polynomial matrix $\boldsymbol{H}(\boldsymbol{z})$ is Laurent polynomial left noninvertible, then $\boldsymbol{H}(\boldsymbol{z})$ is also polynomial left noninvertible. According to Theorem 3, this shows that $\operatorname{RES}_{(P k, \ldots, P k)} \circ \phi(\boldsymbol{H}(\boldsymbol{z}))=0$ for all Laurent polynomial left noninvertible polynomial matrix $\boldsymbol{H}(\boldsymbol{z})$.

\section{Generically Noninvertible when $N-P<M$}

Projective $M$-space $\mathbb{P}^{M}$ is the set of equivalence classes of $(M+1)$-tuples $\left(a_{0}, \ldots, a_{M}\right)$ of elements of $\mathbb{C}$, not all zero, under the equivalence relation given by $\left(a_{0}, \ldots, a_{M}\right) \sim$ $\left(\lambda a_{0}, \ldots ., \lambda a_{M}\right)$ for all nonzero $\lambda \in \mathbb{C}$.

The following lemma depends heavily on commutative ring theory and algebraic geometry. For the details on the definitions of ring, ideal, radical ideal, and prime ideal, please refer to [34] and [35]. For the purpose of our proof, we need only the following definition.

Definition 4 (Height): The height of a prime ideal ht $p$ is the supremum of the lengths $\mathrm{n}$ of strictly descending chains $p=p_{0} \supset p_{1} \supset \ldots \supset p_{n}$ of prime ideals. For an arbitrary ideal $I$, ht $I=\inf \{$ ht $p \mid I \subset p, p$ is prime ideal $\}$.

Lemma 4: Given $\boldsymbol{H}(\boldsymbol{z})$ an $N \times P$ polynomial matrix in $M$ variables of degree at most $k>0$ and $N \geq P$. Let

$$
V\left(\left\{m_{i}\right\}\right):=\left\{\boldsymbol{Z} \in \mathbb{P}^{n} \mid m_{i}(\boldsymbol{Z})=0 \quad \text { for all } i=1, \ldots,\left(\begin{array}{c}
N \\
P
\end{array}\right)\right\}
$$

where $m_{i}$ is a maximal minor of $\overline{\boldsymbol{H}}(\boldsymbol{Z})$ with some ordering and $\bar{H}(\boldsymbol{Z})$ is the homogenization of $\boldsymbol{H}(\boldsymbol{z})$ of degree $k$. Then $V\left(\left\{m_{i}\right\}\right)$ is empty if and only if ht $\left\langle m_{i}\right\rangle=M+1$. Therefore if $V\left(\left\{m_{i}\right\}\right)$ is empty, then $N-P \geq M$. In other words, if $N-P<M$, then $V\left(\left\{m_{i}\right\}\right)$ is nonempty.

Proof: Since $m_{i}$ is homogeneous, then the unit does not lie in $\left\langle m_{i}\right\rangle$. This implies that $\left\langle m_{i}\right\rangle \neq \mathbb{C}\left[x_{0}, \ldots, x_{n}\right]$. By [32, p.370] and the definition of radical ideal, $V\left(\left\{m_{i}\right\}\right)$ is empty if and only if $\sqrt{\left\langle m_{i}\right\rangle}=\left\langle x_{0}, \ldots, x_{M}\right\rangle$. It is easy to see that ht $\sqrt{\left\langle m_{i}\right\rangle}=M+1$. Since ht $\left\langle m_{i}\right\rangle=$ ht $\sqrt{\left\langle m_{i}\right\rangle}$, the height of $\left\langle m_{i}\right\rangle$ is also $M+1$. Macaulay in [36, p.54] proved that ht $\left\langle m_{i}\right\rangle \leq N-P+1$. Therefore if $V\left(\left\{m_{i}\right\}\right)$ is empty, then $N-P \geq M$. In other word, if $N-P<M$, then $V\left(\left\{m_{i}\right\}\right)$ is nonempty.

Definition 5 (Weak-Zero): [29] A point in $\mathbb{P}^{M}$ is said to be weak-zero if at least one of its coordinates is zero.

Lemma 5: A polynomial matrix $\boldsymbol{H}(\boldsymbol{z})$ is Laurent polynomial invertible if and only if the set $V\left(\left\{m_{i}\right\}\right)$ contains only weak-zeros where $\boldsymbol{H}(\boldsymbol{z}), V$ and $m_{i}$ are same as above lemma.

Proof: Follows immediately by Proposition 5.2 in [37].

Remark 3: Suppose now $N-P<M$. By Lemma 4 and Lemma 5, if an $N \times P$ polynomial matrix $\boldsymbol{H}(\boldsymbol{z})$ is Laurent polynomial invertible, then the set $V\left(\left\{m_{i}\right\}\right)$ contains at least one weak-zeros.

Now we can prove the second half of Conjecture 1.

Theorem 5: If $N-P<M$ and $k>0$, then an $N \times P$ polynomial $M$-variate matrix $\boldsymbol{H}(\boldsymbol{z})$ of degree at most $k$ is generically Laurent polynomial left noninvertible.

Proof: The strategy of the proof is the same as in Theorem 3 above. We will find a nonzero polynomial $F$ such that $F(\boldsymbol{H}(\boldsymbol{z}))=0$ for every Laurent polynomial left invertible polynomial matrix $\boldsymbol{H}(\boldsymbol{z})$. 
If $N<P$, then every polynomial matrix is left noninvertible. Thus the statement is true. For example we may arbitrarily set $F=1$. Now we assume $N \geq P$. Suppose $\boldsymbol{H}(\boldsymbol{z})$ is invertible. Since there exists a Laurent polynomial matrix $\boldsymbol{G}(\boldsymbol{z})$ such that $\boldsymbol{G}(\boldsymbol{z}) \boldsymbol{H}(\boldsymbol{z})=\boldsymbol{I}$ and $\boldsymbol{G}\left(z_{1}, \ldots, z_{N-P+1}, 1, \ldots, 1\right)$ is well-defined, $\boldsymbol{H}\left(z_{1}, \ldots, z_{N-P+1}, 1, \ldots, 1\right)$ is also Laurent polynomial invertible. We can now assume that $M=N-P+1$. Define $t_{i}(\boldsymbol{Z})$ to be the same as in the proof of Theorem 3. Let $t_{j}^{(i)}=t_{j}\left(z_{0}, \ldots, \stackrel{i \text {-th }}{0}, \ldots, z_{M}\right)$. For each $i=0, \ldots, M$, define $\theta_{i}$ to be a function such that

$$
\boldsymbol{H}(\boldsymbol{z}) \mapsto\left(t_{0}^{(i)}, \ldots,{\hat{t_{i}}}^{(i)}, \ldots, t_{M}^{(i)}\right)^{T}
$$

where ${\hat{t_{i}}}^{(i)}$ means that the term $t_{i}^{(i)}$ is omitted from the coordinates. By Remark 3 and the fact that $\left\{t_{0}^{(i)}(\boldsymbol{Z}), \ldots,{\hat{t_{i}}}^{(i)}(\boldsymbol{Z}), \ldots, t_{M}^{(i)}(\boldsymbol{Z})\right\} \quad$ is the subset of the set of maximal minors of $\overline{\boldsymbol{H}}(\boldsymbol{Z})$ implies that $\theta_{i}(\boldsymbol{H}(\boldsymbol{z}))$ have a nonzero common zero for some $i=0, \ldots, M$. By the property of the resultant shown in Theorem 2, we know for any Laurent polynomial left invertible polynomial matrix $\boldsymbol{H}(\boldsymbol{z})$ that

$$
\operatorname{RES}_{(P k, \ldots, P k)}\left(\theta_{i}(\boldsymbol{H}(\boldsymbol{z}))\right)=0 \quad \text { for some } i=0, \ldots, M .
$$

Now let

$$
F=\prod_{i=0}^{M} \operatorname{RES}_{(P k, \ldots, P k)} \circ \theta_{i} .
$$

Then $F(\boldsymbol{H}(\boldsymbol{z}))=0$ for all Laurent polynomial left invertible polynomial matrix $\boldsymbol{H}(\boldsymbol{z})$. The $\operatorname{RES}_{(P k, \ldots, P k)}$ and $t_{j}^{(i)}$ are polynomials, so is $F$. Lastly, we need to show $F$ is not a zero function. Let

$$
\boldsymbol{T}(\boldsymbol{z})=\left(\begin{array}{cccc}
1 & 0 & \ldots & 0 \\
z_{1}^{k} & 1 & \ldots & 0 \\
z_{2}^{k} & z_{1}^{k} & \ddots & 0 \\
\vdots & \vdots & \ddots & 1 \\
z_{M}^{k} & z_{M-1}^{k} & \ddots & z_{1}^{k} \\
0 & z_{M}^{k} & \ddots & \vdots \\
\vdots & \vdots & \ddots & \vdots \\
0 & \ldots & 0 & z_{M}^{k}
\end{array}\right)
$$

be an $N \times P$ matrix. Suppose $\operatorname{RES}_{(P k, \ldots, P k)}\left(\theta_{i}(\boldsymbol{T}(\boldsymbol{z}))\right)=0$. By Theorem 2, we know that $\left\{t_{0}^{(i)}, \ldots,{\hat{t_{i}}}^{(i)}, \ldots, t_{M}^{(i)}\right\}$ have a nontrivial common zero. i.e. there exists $\tilde{Z}$ a nonzero solution such that

$$
t_{M}\left(\tilde{z}_{0}, \ldots, \stackrel{i \text {-th }}{0}, \ldots, \tilde{z}_{M}\right)=\tilde{z}_{M}^{P k}=0 .
$$

This implies $\tilde{z}_{M}=0$. If $\tilde{z}_{M}=0$, then

$$
t_{M-1}\left(\tilde{z}_{0}, \ldots, \stackrel{i \text {-th }}{0}, \ldots, \tilde{z}_{M-1}, 0\right)=\tilde{z}_{M-1}^{P k}=0 .
$$

\begin{tabular}{|c|c|c|c|c|c|c|}
\hline & & & \multicolumn{4}{|c|}{$\mathrm{N}$} \\
\hline & & & 1 & 2 & 3 & 4 \\
\hline \multirow{4}{*}{$\mathrm{M}=1$} & & 1 & 0 & 500 & 500 & 500 \\
\hline & $\mathrm{P}$ & 2 & 0 & 0 & 500 & 500 \\
\hline & & 3 & 0 & 0 & 0 & 500 \\
\hline & & 4 & 0 & 0 & 0 & 0 \\
\hline \multirow{4}{*}{$M=2$} & & 1 & 0 & 0 & 500 & 500 \\
\hline & $\mathrm{P}$ & 2 & 0 & 0 & 0 & 500 \\
\hline & & 3 & 0 & 0 & 0 & 0 \\
\hline & & 4 & 0 & 0 & 0 & 0 \\
\hline \multirow{4}{*}{$M=3$} & & 1 & 0 & 0 & 0 & 500 \\
\hline & $\mathrm{P}$ & 2 & 0 & 0 & 0 & 0 \\
\hline & & 3 & 0 & 0 & 0 & 0 \\
\hline & & 4 & 0 & 0 & 0 & 0 \\
\hline
\end{tabular}

Thus $\tilde{z}_{M-1}=0$. Continuing the process, we can conclude $\tilde{z}_{0}=\tilde{z}_{1}=\ldots=\tilde{z}_{M}=0$. This contradicts the assumption that $\tilde{\boldsymbol{Z}}$ is nontrivial. So $\operatorname{RES}_{(P k, \ldots, P k)}\left(\theta_{i}(\boldsymbol{T}(\boldsymbol{z}))\right) \neq 0$ for all $i$. Therefore $F$ is not a zero function. By the definition of hold
TABLE I

INVERSIBILITY TEST FOR A RANDOM POLYNOMIAL MATRIX GENERATOR With DIFFERENT $N, P$ AND $M$ IN 500 TEST CASES

generically, we conclude that $\boldsymbol{H}(\boldsymbol{z})$ of degree at most $k$ is generically polynomial left noninvertible matrix.

Remark 4: Let $\boldsymbol{H}(\boldsymbol{z})$ be a polynomial matrix. If $\boldsymbol{H}(\boldsymbol{z})$ is polynomial left invertible, then $\boldsymbol{H}(\boldsymbol{z})$ is Laurent polynomial left invertible. But the converse is not true in general. Also if $\boldsymbol{H}(\boldsymbol{z})$ is Laurent polynomial left noninvertible, then $\boldsymbol{H}(\boldsymbol{z})$ is polynomial left noninvertible. Also the converse is not true in general.

Example 7: Let $(z)$ be a $1 \times 1$ matrix. It is not polynomial left invertible matrix but it is a Laurent polynomial left invertible matrix as $\left(z^{-1}\right)(z)=1$.

Theorem 6: If $N-P<M$ and $k>0$, then an $N \times P$ polynomial $M$-variate matrix $\boldsymbol{H}(\boldsymbol{z})$ of degree at most $k$ is generically polynomial left noninvertible.

Proof: By Remark 4, we know that if a polynomial matrix $\boldsymbol{H}(\boldsymbol{z})$ is polynomial left invertible, then $\boldsymbol{H}(\boldsymbol{z})$ is also Laurent polynomial left invertible. According to Theorem 5, this shows that $F(\boldsymbol{H}(\boldsymbol{z}))=0$ for all polynomial left invertible polynomial matrix $\boldsymbol{H}(\boldsymbol{z})$.

\section{Simulation and Applications}

We used a random polynomial matrix generator to generate polynomial matrices with each entry of degree less than or equal to 4 and the random coefficients are from 1 to 100. For each value of $N, P$ and $M$, we ran 500 samples to test invertibility. Date from Table I, we shows agreement with our theorems. Another observation was that there was a sharp phase transition from noninvertibility to invertibility depending only the condition $N-P<M$ or $N-P \geq M$.

These theorems lead to some applications. For image deconvolution from multiple FIR blur filters, Harikumar and Bresler in [33], [38] show that perfect reconstruction is almost surely, when there are at least three channels. Since the image is two dimensional (i.e. $M=2$ ) and the downsampling rate is just one (i.e. $P=1$ ), by Theorem 4 , we know that the perfect reconstruction is almost surely if the number of channels is greater than two (i.e. $N \geq 3$ ). Therefore Harikumar and Bresler's image deconvolution is a special case of our main theorem. 
Another application is that we can have an alternative approach in designing multidimensional filter banks. We can freely design the analysis side first such that it satisfies the condition (i.e. $N-P \geq M$ ). Then, by Theorem 4 and Lemma 3 , we can almost surely find a perfect reconstruction inverse for the synthesis polyphase matrix.

\section{FAST COMPUTATION OF LEFT INVERSES}

By Theorem 4, we know we should design the filter banks such that $N-P \geq M$. Suppose $N-P \geq M$. Since $\boldsymbol{H}(\boldsymbol{z})$ is a Laurent polynomial matrix, there exists $\boldsymbol{l} \in \mathbb{N}^{M}$ such that $\boldsymbol{z}^{l} \boldsymbol{H}(\boldsymbol{z})$ is a polynomial matrix and is generically Laurent polynomial left invertible. However, at the same time, the $\boldsymbol{z}^{l} \boldsymbol{H}(\boldsymbol{z})$ is generically polynomial left invertible by Theorem 3. Due to this fact, we can improve our Algorithm 2.

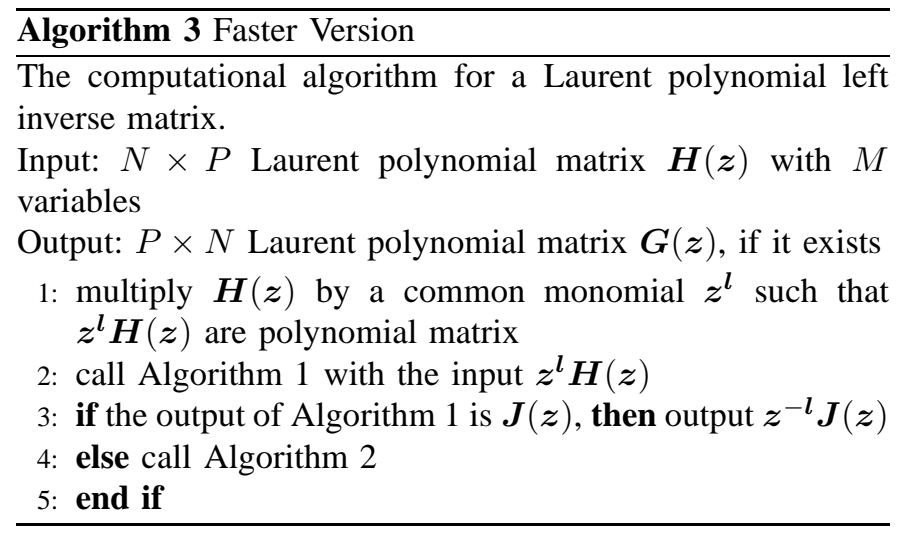

Since Algorithm 1 does not need to introduce any new variable and the matrix is smaller, the computation of Algorithm 1 is faster than Algorithm 2. Moreover, as we mentioned before $\boldsymbol{z}^{l} \boldsymbol{H}(\boldsymbol{z})$ is generically polynomial left invertible, so most of the time we would perform Algorithm 1 in step 3, which leads to less frequent calling of Algorithm 2 in step 4. Therefore, Algorithm 3 is faster than Algorithm 2 in most cases.

Example 8: Compare the processing time between Algorithm 2 and Algorithm 3. Let $\boldsymbol{H}\left(z_{1}, z_{2}\right)$

$$
=\left(\begin{array}{cc}
4 z_{1} & 7 z_{1}{ }^{-1} z_{2}^{2}+2+10 z_{1}{ }^{-1} \\
1+10 z_{1}{ }^{-1} & 10 z_{1}+3 z_{2} \\
7 z_{1}+9 z_{2}+10 z_{1}{ }^{-1} z_{2}+10 z_{1}{ }^{-1} & 0 \\
8 z_{1}{ }^{-1} z_{2}^{2}+10+4 z_{1}{ }^{-1} & 6 z_{1}{ }^{-1} z_{2}^{2}
\end{array}\right)
$$

be a Laurent polynomial matrix. Then let $\boldsymbol{H}^{\prime}\left(z_{1}, z_{2}, w\right)$

$$
=\left(\begin{array}{cc}
4 z_{1}^{2} & 7 z_{2}^{2}+2 z_{1}+10 \\
z_{1}+10 & 10 z_{1}^{2}+3 z_{1} z_{2} \\
7 z_{1}^{2}+9 z_{1} z_{2}+10 z_{2}+10 & 0 \\
8 z_{2}^{2}+10 z_{1}+4 & 6 z_{2}^{2} \\
1-z_{1} z_{2} w & 0 \\
0 & 1-z_{1} z_{2} w
\end{array}\right)
$$

be a polynomial matrix according to Proposition 2 .

To calculate a Laurent polynomial left inverse using Algorithm 2:

$$
\begin{aligned}
& \text { 1: >system("--min-time", "0.02"); } \\
& \text { 2: >timer }=1 ; \quad \triangleright \text { The time of each command is printed } \\
& \text { 3: >int } t=\text { timer; } \quad \triangleright \text { Initialize } t \text { by timer }
\end{aligned}
$$

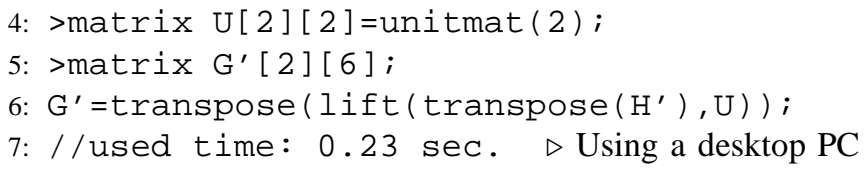
To calculate a Laurent polynomial left inverse using Algorithm 3 in Singular:

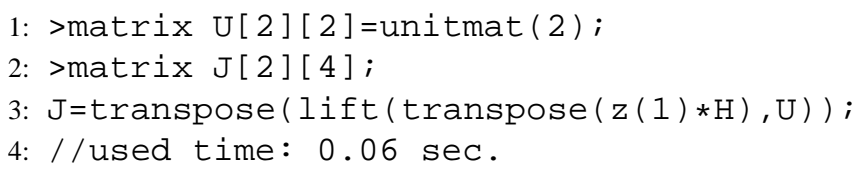

\section{CONCLUSION}

In this paper we study the inverse problem of a Laurent polynomial matrices. Such matrices arise in FIR filter banks as polyphase matrices. We use the computation of Gröbner bases to test the invertibility. Then we propose algorithms to find a particular left inverse. We note that there is a sharp phase transition on the invertibility depending on the size and dimension of a given Laurent polynomial matrix. Specifically when $N-P \geq M$, the $M$-variate $N \times P$ polynomial (resp.: Laurent polynomial) matrix is generically invertible; whereas when $N-P<M$, the matrix is generically noninvertible. Using this sharp phase transition property, we develop a fast algorithm to compute a particular left inverse for a given Laurent polynomial matrix.

These results suggest an alternative approach in designing multidimensional filter banks by freely generating filters for the analysis side first. If we allow an amount of oversampling (i.e. $N-P \geq M$ ), then we can almost surely find a perfect reconstruction inverse for the synthesis polyphase matrix. These results also have potential applications in multidimensional signal reconstruction from multichannel filtering and sampling. Acknowledgment. The authors thank Dr. Jianping Zhou whose $\mathrm{PhD}$ thesis has inspired this work.

\section{REFERENCES}

1] P. P. Vaidyannthan, Multirate Systems and Filter Banks. Prentice-Hall, 1993.

[2] M. Vetterli and J. Kovačević, Wavelets and Subband Coding. PrenticeHall, 1995.

[3] H. Bölcskei, F. Hlawatsch, and H. G. Feichtinger, "Frame-theoretic analysis of oversampled filter banks," IEEE Trans. Signal Proc., vol. 46, pp. 3256-3268, Dec. 1998

[4] Z. Cvetković and M. Vetterli, "Oversampled filter banks," IEEE Trans. Signal Proc., vol. 46, pp. 1245-1255, May. 1998.

[5] J. Kovacevic, P. L. Dragotti, and V. K. Goyal, "Filter bank frame expansions with erasures," IEEE Trans. Information Theory, vol. 48, no. 6, pp. 1439-1450, June 2002.

[6] W. W. Adams and P. Loustaunau, An Introduction to Gröbner Bases. American Mathematical Society, 1994, vol. 3.

[7] D. Cox, J. Little, and D. O'Shea, Using Algebraic Geometry. SpringerVerlag, 1998.

[8] C. Caroenlarpnopparut and N. K. Bose, "Multidimensional FIR filter bank design using Gröbner bases," IEEE Trans. Circ. and Syst., vol. 46, Dec. 1999.

[9] C. Caroenlarpnopparut, "Gröbner bases in multidimensional systems and signal processing," Ph.D. dissertation, Pennsylvania State University, 2000. 
[10] H. Park, T. Kalker, and M. Vetterli, "Gröbner bases and multidimensional FIR multirate systems," Multidimensional Systems and Signal Processing, vol. 8, pp. 11-30, 1997.

[11] H. Park, "Optimal design of synthesis filters in multidimensional perfect reconstruction FIR filter banks using Gröbner bases," IEEE Trans. Circ. and Syst., vol. 49, pp. 843-851, Jun. 2002.

[12] H. Park and G. Regensburger, Eds., Grobner Bases in Control Theory and Signal Processing. Walter de Gruyter, 2007.

[13] L. Zhiping, L. Xu, and N. Bose, "A tutorial on Gröbner Bases with applications in signals and systems," IEEE Trans on Circ. and Syst., vol. 55, no. 1, pp. 445-461, Feb 2008.

[14] R. Rajagopal and L. C. Potter, "Multivariate MIMO FIR inverses," IEEE Trans. Image Proc., vol. 12, pp. 458-465, Apr. 2003.

[15] J. Zhou and M. N. Do, "Multidimensional multichannel FIR deconvolution using Gröbner bases," IEEE Trans. Image Proc., vol. 15, no. 10, pp. 2998-3007, Oct. 2006.

[16] R. Rajagopal and L. C. Potter, "Multi-channel multi-variate equalizer computation," Multidimensional Systems and Signal Processing, vol. 14, pp. 105-108, Jan. 2003.

[17] H. Park, "A computational theory of Laurent polynomial rings and multidimensional FIR systems," Ph.D. dissertation, University of California at Berkeley, 1995.

[18] K. L. Law and M. N. Do, "Multidimensional filter bank signal reconstruction from multichannel acquisition," IEEE Trans. Image Proc., submitted 2008; available at http: //www.ifp.uiuc.edu/ minhdo/publications/.

[19] J. Zhou and M. N. Do, "Multidimensional oversampled filter banks," San Diego, USA: Proc. of SPIE Conference on Wavelet Applications in Signal and Image Processing XI, Jul. 2005.

[20] H. Royden, Real Analysis, 3rd ed. Prentice Hall, 1988.

[21] C. A. Berenstein, A. V. R. Gay, and A. Yger, Residue Currents and Bezout Identities. Birkhäuser, 1993

[22] R. E. Blahut, Fast Algorithms for Digital Signal Processing. AddisonWesley, 1985.

[23] T. Becker and V. Weispfenning, Gröbner Bases. Springer-Verlag, 1993.

[24] B. Buchberger, "Theoretical basis for the reduction of polynomials to canonical forms," ACM SIGSAM Bull, vol. 10, no. 3, pp. 19-29, Aug. 1976.

[25] S. Lang, Algebra. Springer-Verlag, 2002.

[26] G. M. Greuel and G. Pfister, A Singular Introduction to Commutative Algebra. Springer-Verlag, 2002.

[27] D. Eisenbud, Introduction to Commutative Algebra with a View Towards Algebraic Geometry. Springer-Verlag, 1995, vol. 150.

[28] L. Caniglia, G. Cortiñas, S. Danón, J. Heintz, T. Krick, and P. Solern, "Algorithmic aspects of Suslin's proof of Serre's conjecture," Comput Complexity, vol. 3, pp. 31-55, 1993.

[29] J. Zhou, "Multidimensional multirate systems: characterization. design, and applications," Ph.D. dissertation, University of Illinois at UrbanaChampaign, 2005

[30] H. Park, "Complete parametrization of synthesis in multidimensional perfect reconstruction FIR systems," IEEE International Symposium on Circuits and Systems, vol. 5, pp. 41-44, 1999.

[31] R. C. Gunning, Analytic Function of Several Complex Variables. Prentice-Hall, 1965.

[32] D. Cox, J. Little, and D. O'Shea, Ideal, Varieties, and Algorithms. Springer-Verlag, 1996

[33] G. Harikumar and Y. Bresler, "Perfect blind restoration of images blurred by multiple filters:theory and efficient algorithms," IEEE Trans. Image Proc. , vol. 8, no. 2, pp. 202-219, Feb 1999.

[34] H. Matsumura, Commutative Ring Theory. Cambridge University Press, 1986.

[35] R. Hartshorne, Algebraic Geometry. Springer-Verlag, 1977.

[36] F. Macaulay, The Algebraic Theory of Modular Systems. Cambridge University Press, 1916.

[37] E. Fornasini and M. E. Valcher, "Multidimensional systems with finite support behaviors: signal structure, generation, and detection," SIAM Journal on Control and Optimization, vol. 36, no. 2, pp. 760 - 779, Mar. 1998.

[38] G. Harikumar and Y. Bresler, "Exact image deconvolution from multiple FIR blurs," IEEE Trans. Image Proc., vol. 8, no. 6, pp. 846-862, Jun. 1999.

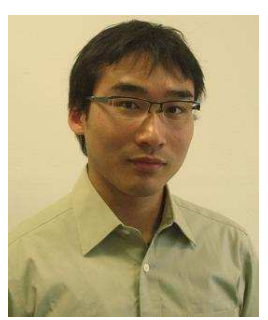

Ka L. Law was born in Hong Kong in 1979. He received the $\mathrm{B}$.Sc. degrees major in computer science from the University of Melbourne, Melbourne, Australia in 2002 and received the B.Sc. degrees in mathematics from the University of Illinois at Urbana-Champaign in 2003. From September 2003 to December 2008, he studied at the Department of Mathematics, the University of Illinois at UrbanaChampaign, where he received the Ph.D. degree in Mathematics with Computational Science and Engineering Option.

He is now with the Department of Communication Systems, Technische Universität, Darmstadt 64283, Germany. His research interests include multidimensional signal processing for FIR filter banks and MIMO systems; adaptive beamforming in MIMO communication systems; convex optimization; and applied algebra and algebraic geometry.

He was a Research Assistant at the Coordinated Science Laboratory, University of Illinois at Urbana-Champaign. He received the Research Assistant Fellowship by Joint Advising Support Program of Applied Mathematics in 2006.

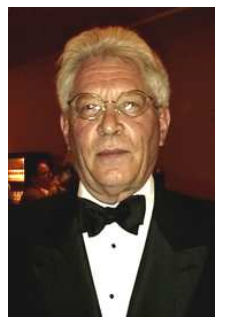

Robert M. Fossum (M'04) was born in Northfield, Minnesota in 1938. He received a B.A degree in mathematics and physics from St. Olaf College in 1959 and $\mathrm{PhD}$ in mathematics from The University of Michigan in 1965

From 1964 to 2008 he was on the faculty of the University of Illinois at Urbana-Champaign in the Department of Mathematics, in the Department of Electrical and Computer Engineering, and jointly with Beckman Institute for Advanced Science and Technology. He is a Professor Emeritus since May

2008.

His research interests are in commutative algebra, algebraic geometry, algebraic groups, and in applications of these mathematical fields to computer vision, image formation and processing, signal processing. He is a foreign member of the Royal Norwegian Society of Sciences and Letters (Det Kongelig Norske Videnskabers Selskab)

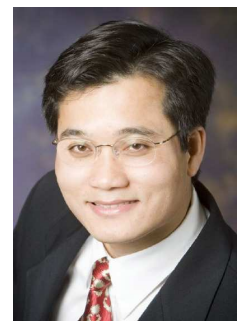

Minh N. Do (M'01, SM'07) was born in Vietnam in 1974. He received the B.Eng. degree in computer engineering from the University of Canberra, Australia, in 1997, and the Dr.Sci. degree in communication systems from the Swiss Federal Institute of Technology Lausanne (EPFL), Switzerland, in 2001.

Since 2002, he has been on the faculty at the University of Illinois at Urbana-Champaign, where he is currently an Associate Professor in the Department of Electrical and Computer Engineering, and hold joint appointments with the Coordinated Science Laboratory an the Beckman Institute for Advanced Science and Technology. His research interests include image and multi-dimensional signal processing, wavelets and multiscale geometric analysis, computational imaging, and visual information representation.

He received a Silver Medal from the 32nd International Mathematical Olympiad in 1991, a University Medal from the University of Canberra in 1997, a Doctorate Award from the EPFL in 2001, a CAREER Award from the National Science Foundation in 2003, and a Young Author Best Paper Award from IEEE in 2008. He was named a Beckman Fellow at the Center for Advanced Study, UIUC, in 2006, and received of a Xerox Award for Faculty Research from the College of Engineering, UIUC, in 2007. He is a member of the IEEE Signal Processing Theory and Methods and Image, Video, and Multidimensional Signal Processing Technical Committees, and an Associate Editor of the IEEE Transactions on Image Processing. 